\title{
Adherence to Mediterranean diet reduces incident frailty risk: A systematic review and meta-analysis
}

Gotaro Kojima, $\mathrm{MD}^{1}$; Christina Avgerinou, $\mathrm{PhD}^{1}$; Steve Iliffe, $\mathrm{FRCGP}^{1}$; Kate Walters, $\mathrm{PhD}^{1}$.

${ }^{1}$ Department of Primary Care and Population Health, University College London, London, UK

\begin{abstract}
Objectives:To conduct a systematic review of the literature on prospective cohort studies examining associations between adherence to a Mediterranean diet and incident frailty, and to perform a meta-analysis to synthesize the pooled risk estimates.
\end{abstract}

Design:Systematic review and meta-analysis.

Setting:Embase, MEDLINE, CINAHL, PsycINFO and Cochrane Library were systematically searched on 14th September 2017. We reviewed references of included studies and relevant review papers and performed a forward citation tracking for additional studies. Corresponding authors were contacted for additional data necessary for a meta-analysis. Participants:Community-dwelling older people with mean age of 60 years and older.

Measurements:Incident frailty risk according to adherence to a Mediterranean diet.

Results:Two reviewers independently screened the title, abstract and full-text to ascertain the eligibility of 125 studies identified by the systematic search of the literature, and four studies were included (5,789 older people with mean follow-up of 3.9 years). Data were extracted from the studies by two reviewers independently. All four studies provided adjusted odds ratios of incident frailty risk according to three Mediterranean Diet Score (MDS) groups (0-3, 4-5 and 6-9). Compared with low adherence to a Mediterranean diet (MDS 0-3), higher adherence was associated with significantly lower incident frailty risks (pooled $\mathrm{OR}=0.62$, $95 \% \mathrm{CI}=0.47-0.82, \mathrm{p}=0.001$ for $\mathrm{MDS} 4-5$; pooled $\mathrm{OR}=0.44,95 \% \mathrm{CI}=0.31-0.64, \mathrm{p}<0.001$ for MDS 6-9). Neither significant heterogeneity $\left(\mathrm{I}^{2}=0-16 \%, \mathrm{p}>0.30\right)$ nor evidence of publication bias was observed.

Conclusion: Greater adherence to a Mediterranean diet is associated with a significantly lower risk of incident frailty among community-dwelling older people. Future studies should confirm these findings and evaluate whether adherence to a Mediterranean diet can reduce the risk of frailty, including in non-Mediterranean populations.

Keywords: Frailty; Mediterranean diet; Systematic review; Meta-analysis. 


\section{INTRODUCTION}

Frailty is a geriatric syndrome common among older people and its prevalence is considered to increase with population ageing. ${ }^{1}$ Although consensus has not been reached on a gold standard definition, frailty is generally defined as a state of increased vulnerability due to age-related accumulation of deficits and decreased physiological reserves across multiple systems. ${ }^{1}$ Frailty predicts numerous negative health outcomes among older people, including falls, ${ }^{2}$ fractures,${ }^{3}$ hospitalization, ${ }^{4}$ nursing home placement,${ }^{5}$ disability, ${ }^{6}$ dementia ${ }^{7}$ and premature death, ${ }^{8}$ and is associated with lower quality of life. ${ }^{9}$

Nutrition is considered to play a crucial role in the complex pathogenesis of frailty. ${ }^{10}$ Nutrition provides energy and essential nutrients, and helps the human body to function properly and maintain homeostasis. The nutrients reported by observational and intervention studies as showing promising results in relation to frailty are protein and some selected micronutrients, such as carotenoids and vitamins. ${ }^{11,12}$ Although evidence on individual nutrients or food items is important, when the food is consumed various micro- and macronutrients interact with each other and synergistic effects may occur. Therefore, instead of focusing on a single dietary component it may be more reasonable to describe the overall consumption of a diet as a dietary pattern. ${ }^{13}$

The Mediterranean diet is based on food patterns typical of Greece and Southern Italy in the 1960s. This dietary pattern, which is traditionally presented as a food pyramid, ${ }^{14}$ consists of abundant plant foods (fruit, vegetable, cereals, potatoes, beans, nuts and seeds), olive oil as the principal source of fat, dairy products, fish and poultry consumed in low to moderate amounts, zero to four eggs consumed weekly, red meat consumed in low amounts, and wine consumed in low to moderate amounts, normally with meals. This diet is low in saturated fat and has been associated with multiple health benefits, including lower incidence of cardiovascular disease, neurodegenerative diseases, diabetes, and overall cancer incidence, as well as prolonged survival ${ }^{15-17}$. So far only a few studies have examined associations between the Mediterranean diet and frailty risks, and the results have been mixed and inconclusive. ${ }^{11,13}$ Furthermore, to our knowledge no meta-analysis has been carried out. The objectives of the current study were thus to conduct a systematic review of the literature on prospective cohort studies examining associations between adherence to a Mediterranean diet and incident frailty, and to perform a meta-analysis to synthesize the pooled risk estimates.

\section{METHOD}

\section{Data source and search strategy}

A systematic search of the literature was conducted on 14th September 2017, based on a protocol generated in accordance with the PRISMA guidelines for systematic reviews. ${ }^{18}$ The five databases used were Embase, MEDLINE, CINAHL Plus, PsycINFO and Cochrane Library (supplementary Table 1). We searched for studies published in 2000 or later, as the most commonly used frailty phenotype criteria were first published in 2001. ${ }^{19}$ The search terms used were a combination of Medical Subject Heading (MeSH) and text terms, including "Mediterranean diet" (MeSH) OR "Mediterranean*" AND "Frailty Syndrome" $(\mathrm{MeSH}) \mathrm{OR}$ "frail*". We used explosion functions where available and did not restrict the search by language. We reviewed references of included studies and relevant review papers and performed a forward citation tracking with Google Scholar for additional studies. We also contacted corresponding authors for additional data necessary for a meta-analysis.

\section{Study selection}

Two reviewers (GK and CA) independently screened the title, abstract and full-text to 
ascertain the eligibility of the studies identified by the literature search. Disagreements were resolved by discussion. Studies were included if they met the following inclusion criteria:

- Involved community-dwelling older people with mean age of 60 and older.

- Assessed adherence to a Mediterranean diet using a tool, such as Mediterranean Diet Score (MDS). ${ }^{17}$

- Prospectively examined risk of developing frailty, defined by original or modified version of validated criteria designed to define frailty, according to adherence to a Mediterranean diet at baseline.

- Provided odds ratio (OR), hazard ratio (HR) or risk ratio (RR) as a risk, or sufficient information to calculate these risk estimates.

Studies were excluded by applying the following exclusion criteria:

- Defined frailty by a single factor (e.g. being certified for long-term care insurance) or by an individual component of frailty criteria (e.g. slow walking speed).

- Used selected cohorts with specific diseases or conditions.

- Review papers, randomized controlled trials, conference abstracts, comments, or editorials.

\section{Methodological quality assessment}

We used the 9-item Newcastle-Ottawa scale for cohort studies to assess methodological quality of the eligible studies. ${ }^{20}$ A study was considered to have adequate methodological quality when the study met five criteria or more out of nine.

\section{Data extraction}

The following data were extracted from the included studies independently by two reviewers (GK and CA): first author, publication year, cohort name if any, location, sample size, proportion of female participants, mean age, age range, tool used to measure adherence to a Mediterranean diet, frailty criteria, and follow-up period. We also extracted risk estimates along with $95 \%$ confidence intervals $(95 \% \mathrm{CI})$ of the final models and covariates used for adjustment.

\section{Statistical analysis}

A meta-analysis was attempted where there were three or more studies providing the same type of effect measures of frailty risk and where adherence to a Mediterranean diet was based on the same tool. Adjusted effect measures were preferred to unadjusted ones when a study reported both. The presence and degree of heterogeneity was assessed using a chi square test and $\mathrm{I}^{2}$ statistic, respectively, and the $\mathrm{I}^{2}$ values of $25 \%, 50 \%$, and $75 \%$ were considered as low, moderate, and high degrees of heterogeneity, respectively. ${ }^{21}$ A fixed-effects model was used if heterogeneity was absent, or a random-effects model was used if heterogeneity was present, to calculate a pooled risk estimate using the generic inverse variance method. Publication bias was assessed by visual inspection of funnel plots. If significant heterogeneity was observed, sensitivity analysis, subgroup analysis and random-effects meta-regression analysis would be performed to further explore the cause of the heterogeneity. Review Manager 5 (version 5.2, The Cochrane Collaboration, Copenhagen, Denmark) was used for all analyses, and the level of significance was set at a $\mathrm{p}$ value of less than 0.05 .

\section{RESULTS}

\section{Selection Process and Study Characteristics}

Figure 1 presents the PRISMA flowchart of the study selection process. Of a total of 125 studies identified, four studies were included $(5,789$ older people with a mean follow-up of 
3.9 years). All studies met five or more of nine items of the Newcastle-Ottawa scale (mean 8.0, range 7-9, Supplementary table 2). Characteristics of the four included studies are summarized in Table 1. MDS was used by all four studies. MDS is a tool to measure adherence to the traditional Mediterranean diet based on the salient characteristic of this diet, ranging 0 to 9 with a higher score indicative of higher adherence. ${ }^{17}$ Three studies $^{22-24}$ used modified versions of the Cardiovascular Health Study frailty criteria, which is one of the most widely measures to define physical frailty based on five criteria (unintentional weight loss, self-reported exhaustion, weakness, slow walking speed and low physical activity). ${ }^{19}$ One study ${ }^{25}$ used FRAIL scale, which can measure frailty by answering five simple questions about fatigue, resistance, ambulation, illnesses and loss of weight. ${ }^{26}$ Two studies divided the cohort into three groups (MDS of 0-3, 4-5 and 6-9), ${ }^{23,24}$ and one study used gender-stratified tertiles. ${ }^{22}$ Two studies used MDS as a continuous variable. ${ }^{24,25}$ All four of the studies included provided ORs of incident frailty risks and used MDS to measure adherence to a Mediterranean diet. ${ }^{22-25}$ One of the four studies showed results using Mediterranean Diet Adherence Screener (MEDAS) as well. ${ }^{22}$ Therefore, ORs according to MDS were chosen for the meta-analysis. All ORs were adjusted for multiple confounders, which include age, gender, education, marital status, body mass index, smoking, alcohol, living status, energy intake, medication, physical activity and comorbidities (Table 1).

\section{Adherence to a Mediterranean Diet and Risk of Incident Frailty}

Odds ratios of incident frailty risk according to three MDS groups (0-3, 4-5 and 6-9) were available from four studies, ${ }^{22-25}$ and used for a fixed-effects meta-analysis since no significant heterogeneity $\left(\mathrm{I}^{2}=0 \%, \mathrm{p}=0.84 ; \mathrm{I}^{2}=16 \%, \mathrm{p}=0.31\right.$, respectively) was observed. The forest plots are shown in Figure 2. Compared with low adherence to a Mediterranean diet (MDS 0-3), higher adherence was associated with significantly lower incident frailty risks (four studies: pooled $\mathrm{OR}=0.62,95 \% \mathrm{CI}=0.47-0.82, \mathrm{p}=0.001$ for $\mathrm{MDS} 4-5$; pooled $\mathrm{OR}=0.44,95 \% \mathrm{CI}=0.31$ $0.64, p<0.001$ for MDS 6-9). No obvious asymmetry was observed in the funnel plots for either analysis. We repeated meta-analyses (1) using a random-effect model (four studies: pooled $\mathrm{OR}=0.62,95 \% \mathrm{CI}=0.47-0.82, \mathrm{p}=0.001$ for $\mathrm{MDS} 4-5$; pooled $\mathrm{OR}=0.44,95 \% \mathrm{CI}=0.29$ $0.66, p<0.001$ for MDS 6-9), and (2) excluding the Chinese study ${ }^{25}$ (three studies: pooled $\mathrm{OR}=0.60,95 \% \mathrm{CI}=0.44-0.82, \mathrm{p}=0.001$ for $\mathrm{MDS} 4-5$; pooled $\mathrm{OR}=0.40,95 \% \mathrm{CI}=0.27-0.65$, $\mathrm{p}<0.001$ for MDS 6-9). This made little difference to our findings. Subgroup analysis and meta-regression analysis were not performed due to the low degree of heterogeneity and the small number of included studies.

\section{DISCUSSION}

This systematic review and meta-analysis identified four studies, including a total of 5,789 community-dwelling older people followed up over a mean period of 3.9 years, and found higher adherence to a Mediterranean diet to be significantly associated with a lower risk of incident frailty.

Adherence to a Mediterranean diet was measured using $\operatorname{MDS}^{17}$ in all the included studies. This is based on the intake of food items specific to the traditional Mediterranean dietary pattern: high intake of fruits, vegetables, legumes, nuts, cereals, fish and olive oil (coupled with low intake of saturated fats), low intake of meat and dairy products, and regular but moderate intake of alcohol, mostly wine. ${ }^{17}$ The beneficial effects of a Mediterranean dietary pattern on frailty we observed is consistent with findings from a cross-sectional study of 923 older Taiwanese people, which explored 79 food items to find the dietary pattern most protective against frailty using reduced rank regression analysis. ${ }^{27}$ In the dietary pattern identified as best for explaining frailty status, fresh fruit, nuts and seeds, vegetables, whole 
grains, fish and breakfast cereals were among the top food items demonstrating protective effects against frailty (factor loading value $=-0.48,-0.39,-0.33,-0.27,-0.20$ and -0.17 , respectively). ${ }^{27}$ Olive oil and wine were not included, perhaps because they were not commonly consumed by this non-Mediterranean population.

Besides Mediterranean diet, other dietary patterns have been investigated and found to be associated with future frailty risks. A "Prudent dietary pattern" characterized by the high consumption of olive oil, vegetables, potatoes, legumes, blue fish, pasta and meat was identified by principal component analysis and was shown to be associated with lower risk of incident frailty over a 3.5-year follow-up in 1872 Spanish older people. ${ }^{28}$ Another study used the Three-City Study Bordeaux cohort of 972 older people and showed that a "healthy" cluster defined by a hybrid clustering method, which is associated with high intake of fish in men and fruits and vegetables in women, was associated with lower risk of developing frailty during a long follow-up of 12 years. ${ }^{29}$ Interestingly, a study from the Netherlands found that high adherence to a "Traditional" dietary pattern, characterized by a high intake of savory snacks, legumes, eggs, fried potatoes, alcohol, processed meat and soup, and in a culturally different setting, was also associated with less frailty defined by the Frailty Index four years later. ${ }^{30}$ This effect was observed despite this dietary pattern including foods such as fried potatoes and processed meat, which are associated with higher incident of cardiovascular disease. Three studies examined associations between quality of diet, using the Dietary Quality Index (based on dietary variety, adequacy of vegetable, fruit, grain, fiber, protein, iron, calcium and vitamin C, moderation of total fat, saturated fat, cholesterol, sodium and empty calorie foods and overall balance of macronutrient ratio and fatty acid ratio) $)^{25,31}$ and the Dutch Healthy Diet index (based on vegetable, fruit, fiber, fish and fish oil, saturated fatty acid, trans-fatty acid, sodium and alcohol), ${ }^{30}$ and subsequent frailty risks and found that higher dietary quality was consistently associated with lower future frailty risks. These findings are, at least partially, in line with ours in that some dietary components are in common with a Mediterranean diet, such as olive oil, vegetables, fruits, legumes and fish. This study's strengths include its robust methodology, including a comprehensive and reproducible search strategy following PRISMA guidelines. ${ }^{18}$ Two authors independently reviewed the search results and extracted the data. In addition, methodological quality, heterogeneity and publication bias were assessed. We were able to include all four of the studies in the meta-analysis by obtaining additional data from the authors of the original papers. $^{22,25}$ All four studies have adequate methodological quality and there is low degree of heterogeneity in their findings. Furthermore, all the effect measures combined in the metaanalysis were fully adjusted for multiple confounders, including age, gender and education. These should contribute to robustness of our synthesized results. However, the current study is not without limitations. Only a small number $(n=4)$ of studies were identified, probably because frailty research in relation to diet has only recently emerged. Further studies are warranted to elucidate associations between Mediterranean diet and frailty, in particular in non-Mediterranean countries. In addition further information on which components of the Mediterranean diet are associated with frailty (e.g. fruit and vegetable, red wine) and which components of frailty are most affected (e.g. measure of muscle strength, exhaustion or weight loss) would give further insight. Adherence to a Mediterranean diet was measured by MDS in all the studies included. Although MDS may be a good indicator of adherence to a Mediterranean diet in Mediterranean populations, its relevance to non-Mediterranean populations is contested. ${ }^{32}$ Some of the MDS components were based on actual food consumption with cut-points specific to the study population (e.g. median value of the intake), rather than based on the intake recommended in a traditional Mediterranean diet. ${ }^{17}$ Therefore the findings based on MDS may not reflect true adherence to a Mediterranean 
diet ${ }^{33}$ especially in non-Mediterranean populations. ${ }^{25}$ Lastly there may be potentially unmeasured confounding as not all studies adjusted for factors related to healthier lifestyle in general, such as smoking, alcohol or physical activity.

There are several potential mechanisms underlying the association between greater adherence to a Mediterranean diet and lower risks of frailty. One possibility is the high intake of foods rich in anti-oxidants. Fruit and vegetables are rich in carotenoids and vitamins and red wine contains abundant polyphenols. Oxidative stress is a risk factor for frailty, ${ }^{34}$ and fruit and vegetables rich in anti-oxidants may decrease the risk of frailty by counteracting oxidative status. Inflammation is another possible explanation. It is known that frail individuals have higher levels of inflammatory markers, and inflammation is considered to be closely associated with frailty. ${ }^{35}$ A Mediterranean diet is associated with low levels of inflammatory markers ${ }^{36}$ and may reduce frailty risk through this mechanism. Adherence to a Mediterranean diet has been associated with better cognitive function, lower rates of cognitive decline and lower risks of Alzheimer's disease and dementia. ${ }^{37,38}$ Moreover, Mediterranean diet has been associated with lower incidence of cardiovascular disease $\mathrm{e}^{39}$ and certain types of cancers, such as colorectal cancer. ${ }^{40}$ All of the above may contribute to the accumulation of fewer health deficits over time, thus resulting in lower incidence of frailty.

\section{CONCLUSION}

This systematic review and meta-analysis shows the first pooled evidence that greater adherence to a Mediterranean diet is associated with a significantly lower risk of incident frailty among community-dwelling older people. Related topics warranting future research include a focus on which components or combination of food contents contribute to the improvement of frailty. We now also need studies to confirm these findings and determine if increasing adherence to a Mediterranean diet can reduce the risk of frailty, including in nonMediterranean populations.

\section{ACKNOWLEGMENT}

We thank authors of the original studies who shared additional data. ${ }^{22,25}$

\section{Conflict of Interest}

The authors have no conflicts in the cover letter as well as in the manuscript, as noted above.

\section{Author Contributions}

Study concept and design: GK, SI and KW. Acquisition of data: GK and CA. Analysis and interpretation of data: GK, CA, SI and KW. Drafting the article: GK. Revising the article critically for important intellectual content: CA, SI and KW. Final approval of the version to be published: GK, CA SI and KW.

\section{Sponsor's Role}

None

\section{REFERENCES}

1. Clegg A, Young J, Iliffe S, et al. Frailty in elderly people. Lancet 2013;381:752-762.

2. Kojima G. Frailty as a Predictor of Future Falls Among Community-Dwelling Older People: A Systematic Review and Meta-Analysis. J Am Med Dir Assoc 2015;16:1027-1033.

3. Kojima G. Frailty as a predictor of fractures among community-dwelling older 
people: A systematic review and meta-analysis. Bone 2016;90:116-122.

4. Kojima G. Frailty as a predictor of hospitalisation among community-dwelling older people: a systematic review and meta-analysis. J Epidemiol Community Health 2016;70:722-729.

5. Kojima G. Frailty as a Predictor of Nursing Home Placement among CommunityDwelling Older Adults: A Systematic Review and Meta-analysis. J Geriatr Phys Ther 2016;

6. Kojima G. Frailty as a predictor of disabilities among community-dwelling older people: a systematic review and meta-analysis. Disabil Rehabil 2017;39:1897-1908.

7. Kojima G, Taniguchi Y, Iliffe S, et al. Frailty as a Predictor of Alzheimer Disease, Vascular Dementia, and All Dementia Among Community-Dwelling Older People: A Systematic Review and Meta-Analysis. J Am Med Dir Assoc 2016;17:881-888.

8. Kojima G, Iliffe S, Walters K. Frailty Index as a Predictor of Mortality: A Systematic Review and Meta-analysis. Age Ageing 2017;

9. Kojima G, Iliffe S, Jivraj S, et al. Association between frailty and quality of life among community-dwelling older people: a systematic review and meta-analysis. J Epidemiol Community Health 2016;70:716-721.

10. Goisser S, Guyonnet S, Volkert D. The Role of Nutrition in Frailty: An Overview. J Frailty Aging 2016;5:74-77.

11. Yannakoulia M, Ntanasi E, Anastasiou CA, et al. Frailty and nutrition: From epidemiological and clinical evidence to potential mechanisms. Metabolism 2017;68:64-76.

12. Morley JE, Vellas B, van Kan GA, et al. Frailty consensus: a call to action. J Am Med Dir Assoc 2013;14:392-397.

13. MacDonell SO, Miller JC, Waters DL, et al. Dietary Patterns in the Frail Elderly. Current Nutrition Reports 2016;5:68-75.

14. Willett WC, Sacks F, Trichopoulou A, et al. Mediterranean diet pyramid: a cultural model for healthy eating. Am J Clin Nutr 1995;61:1402S-1406S.

15. Sofi F, Cesari F, Abbate R, et al. Adherence to Mediterranean diet and health status: meta-analysis. BMJ 2008;337:a1344.

16. Dinu M, Pagliai G, Casini A, et al. Mediterranean diet and multiple health outcomes: an umbrella review of meta-analyses of observational studies and randomised trials. Eur J Clin Nutr 2017;

17. Trichopoulou A, Costacou T, Bamia C, et al. Adherence to a Mediterranean diet and survival in a Greek population. N Engl J Med 2003;348:2599-2608.

18. Moher D, Liberati A, Tetzlaff J, et al. Preferred reporting items for systematic reviews and meta-analyses: the PRISMA statement. BMJ 2009;339:b2535.

19. Fried LP, Tangen CM, Walston J, et al. Frailty in older adults: evidence for a phenotype. J Gerontol A Biol Sci Med Sci 2001;56:M146-156.

20. Wells GA, Shea D, O'Connell D, et al. The Newcastle-Ottawa Scale (NOS) for assessing the quality of nonrandomised studies in meta-analyses. http://www.ohri.ca/programs/clinical_epidemiology/oxford.asp. Accessed 20th August, 2015.

21. Higgins JP, Thompson SG, Deeks JJ, et al. Measuring inconsistency in meta-analyses. BMJ 2003;327:557-560.

22. Leon-Munoz LM, Guallar-Castillon P, Lopez-Garcia E, et al. Mediterranean diet and risk of frailty in community-dwelling older adults. J Am Med Dir Assoc 2014;15:899 903.

23. Talegawkar SA, Bandinelli S, Bandeen-Roche K, et al. A higher adherence to a Mediterranean-style diet is inversely associated with the development of frailty in 
community-dwelling elderly men and women. J Nutr 2012;142:2161-2166.

24. Rahi B, Ajana S, Tabue-Teguo M, et al. High adherence to a Mediterranean diet and lower risk of frailty among French older adults community-dwellers: Results from the Three-City-Bordeaux Study. Clin Nutr 2017;

25. Chan R, Leung J, Woo J. Dietary Patterns and Risk of Frailty in Chinese CommunityDwelling Older People in Hong Kong: A Prospective Cohort Study. Nutrients 2015;7:7070-7084.

26. Morley JE, Malmstrom TK, Miller DK. A simple frailty questionnaire (FRAIL) predicts outcomes in middle aged African Americans. J Nutr Health Aging 2012;16:601-608.

27. Lo YL, Hsieh YT, Hsu LL, et al. Dietary Pattern Associated with Frailty: Results from Nutrition and Health Survey in Taiwan. J Am Geriatr Soc 2017;65:2009-2015.

28. Leon-Munoz LM, Garcia-Esquinas E, Lopez-Garcia E, et al. Major dietary patterns and risk of frailty in older adults: a prospective cohort study. BMC Med 2015;13:11.

29. Pilleron S, Ajana S, Jutand MA, et al. Dietary Patterns and 12-Year Risk of Frailty: Results From the Three-City Bordeaux Study. J Am Med Dir Assoc 2017;18:169-175.

30. de Haas SCM, de Jonge EAL, Voortman T, et al. Dietary patterns and changes in frailty status: the Rotterdam study. Eur J Nutr 2017;

31. Shikany JM, Barrett-Connor E, Ensrud KE, et al. Macronutrients, diet quality, and frailty in older men. J Gerontol A Biol Sci Med Sci 2014;69:695-701.

32. Hoffman R, Gerber M. Evaluating and adapting the Mediterranean diet for nonMediterranean populations: a critical appraisal. Nutr Rev 2013;71:573-584.

33. Rumawas ME, Dwyer JT, McKeown NM, et al. The development of the Mediterranean-style dietary pattern score and its application to the American diet in the Framingham Offspring Cohort. J Nutr 2009;139:1150-1156.

34. Soysal P, Isik AT, Carvalho AF, et al. Oxidative stress and frailty: A systematic review and synthesis of the best evidence. Maturitas 2017;99:66-72.

35. Soysal P, Stubbs B, Lucato P, et al. Inflammation and frailty in the elderly: A systematic review and meta-analysis. Ageing Res Rev 2016;31:1-8.

36. Casas R, Sacanella E, Estruch R. The immune protective effect of the Mediterranean diet against chronic low-grade inflammatory diseases. Endocr Metab Immune Disord Drug Targets 2014;14:245-254.

37. Lourida I, Soni M, Thompson-Coon J, et al. Mediterranean diet, cognitive function, and dementia: a systematic review. Epidemiology 2013;24:479-489.

38. Singh B, Parsaik AK, Mielke MM, et al. Association of mediterranean diet with mild cognitive impairment and Alzheimer's disease: a systematic review and meta-analysis. J Alzheimers Dis 2014;39:271-282.

39. Liyanage T, Ninomiya T, Wang A, et al. Effects of the Mediterranean Diet on Cardiovascular Outcomes-A Systematic Review and Meta-Analysis. PLoS One 2016;11:e0159252.

40. Farinetti A, Zurlo V, Manenti A, et al. Mediterranean diet and colorectal cancer: A systematic review. Nutrition 2017;43-44:83-88. 
Table 1. Summary of included studies on Mediterranean diet and incident frailty.

\begin{tabular}{|c|c|c|c|c|c|c|c|c|}
\hline $\begin{array}{l}\text { Author/Year } \\
\text { Cohort name }\end{array}$ & Location & $\begin{array}{l}\text { Sample } \\
\text { size }\end{array}$ & $\begin{array}{c}\text { Female } \\
(\%)\end{array}$ & Age (range) & $\begin{array}{c}\text { Mediterranean diet } \\
\text { measurement }\end{array}$ & $\begin{array}{l}\text { Frailty } \\
\text { criteria }\end{array}$ & $\begin{array}{c}\text { Follow-up } \\
\text { period }\end{array}$ & NOS \\
\hline $\begin{array}{l}\text { Rahi } 2017 \\
\text { Three-City Study }\end{array}$ & France & 560 & $63.2 \%$ & $\begin{array}{l}81.7 \\
(\geq 75)\end{array}$ & MDS & mCHS & 2 years & $9 / 9$ \\
\hline Chan 2015 & China & 2724 & $50.3 \%$ & $\begin{array}{l}71.8 \\
(\geq 65)\end{array}$ & MDS & FRAIL & 4 years & $8 / 9$ \\
\hline $\begin{array}{l}\text { Leon-Munoz } 2014 \\
\text { Seniors-ENRICA }\end{array}$ & Spain & 1815 & - & $(>60)$ & $\begin{array}{c}\text { MDS } \\
\text { MEDAS }\end{array}$ & $\mathrm{mCHS}$ & 3.5 years & $8 / 9$ \\
\hline $\begin{array}{l}\text { Talegawkar } 2012 \\
\text { InCHIANTI }\end{array}$ & Italy & 690 & $51.7 \%$ & $\begin{array}{l}73.0 \\
(\geq 60)\end{array}$ & MDS & mCHS & 6 years & $7 / 9$ \\
\hline
\end{tabular}

$95 \% \mathrm{CI}=95 \%$ confidence interval

aOR: Adjusted odds ratio

mCHS: Modified Cardiovascular Health Study frailty criteria

MEDAS: Mediterranean Diet Adherence Screener

MDS: Mediterranean diet score (range: 0-9)

NOS: Newcastle-Ottawa Scale (range: 0-9)

Seniors-ENRICA: Study on Nutrition and Cardiovascular Risk Factors in Spain 
Figure 1. Flow chart of systematic literature review.

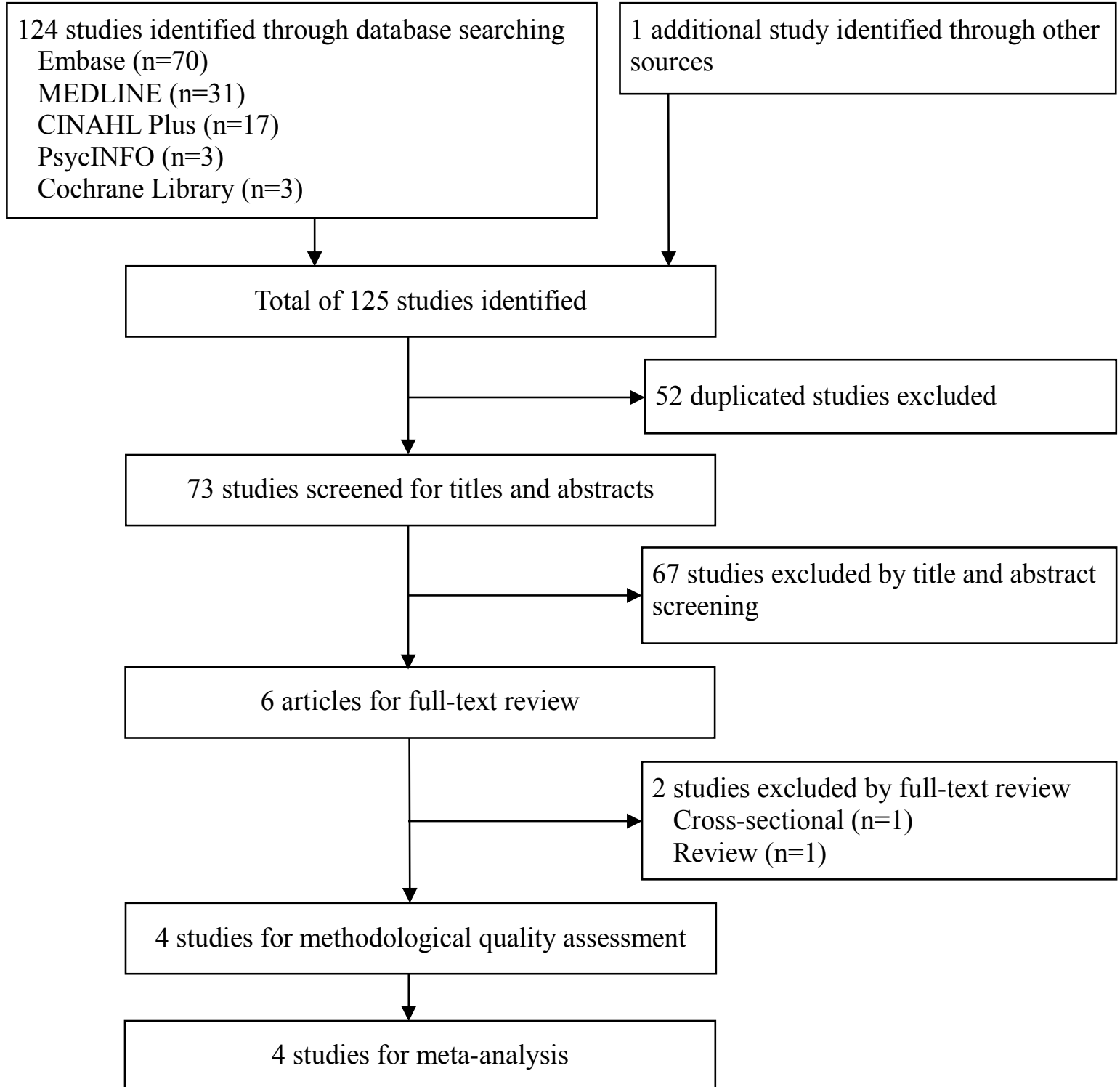


Figure 2. Forest plots of incident frailty risk according to Mediterranean diet score. (MDS 4-5 vs. MDS 0-3; MDS 6-9 vs. MDS 0-3)

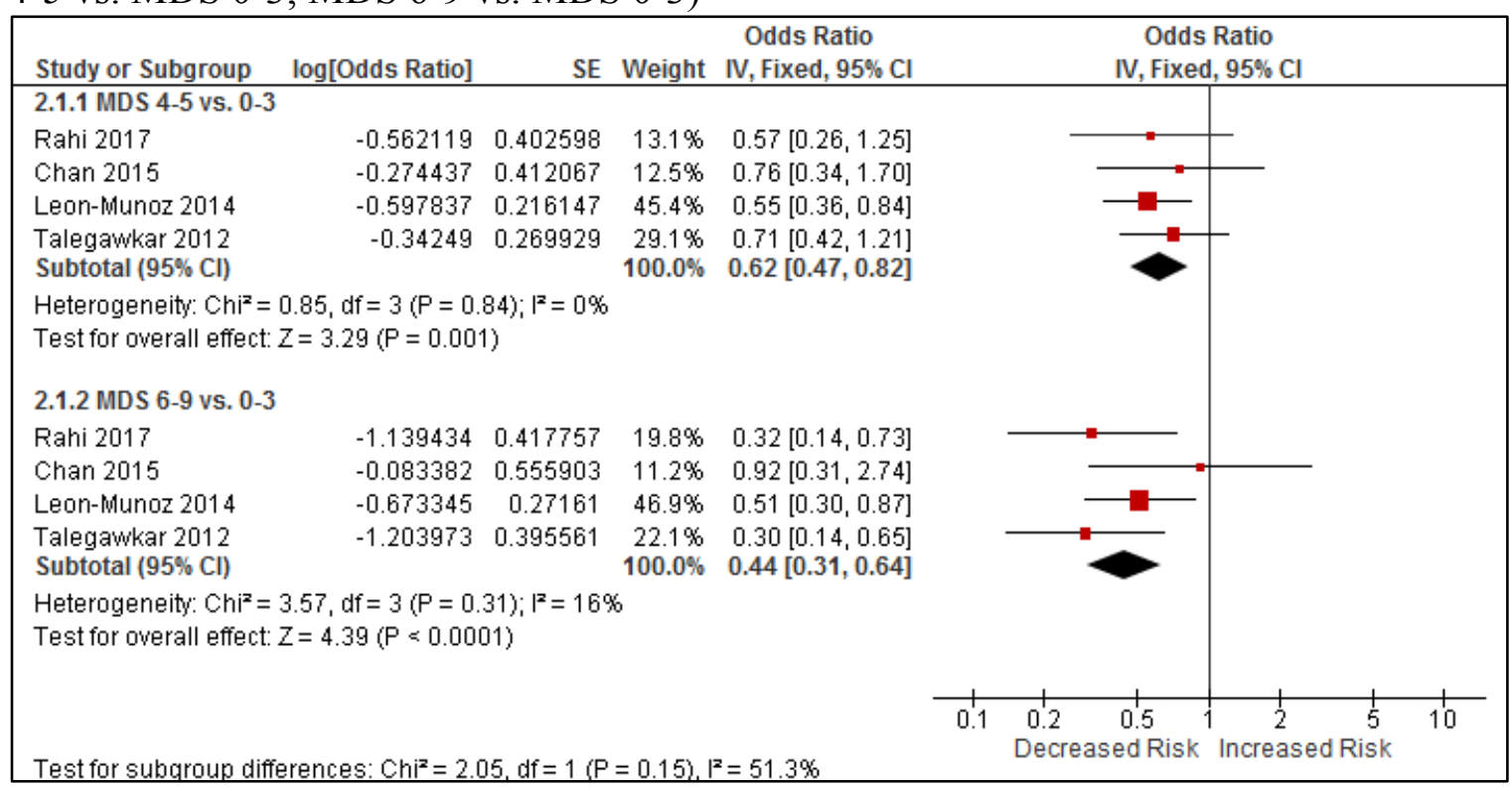

